\title{
An fMRI Study to Analyze Neural Correlates of Presence during Virtual Reality Experiences
}

\author{
Miriam Clemente ${ }^{1}$, Beatriz Rey ${ }^{1,2}$, Aina Rodríguez-Pujadas ${ }^{3}$, Alfonso $^{2}$ \\ Barros-Loscertales ${ }^{3}$, Rosa M. Baños ${ }^{2,4}$, Cristina Botella ${ }^{2,5}$, Mariano \\ AlCAÑIZ ${ }^{1,2}$, AND CÉSAR ÁvILA ${ }^{3}$ \\ ${ }^{1}$ I3BH/LabHuman, Instituto Interuniversitario de Investigación en Bioingeniería y Tecnología orientada al \\ Ser Humano, Universitat Politècnica de València, Valencia, Spain \\ ${ }^{2}$ Ciber, Fisiopatología Obesidad y Nutrición, CB06/03 Instituto de Salud Carlos III, Spain \\ ${ }^{3}$ Departamento de Psicología Básica, Clínica y Psicobiología, Universitat Jaume I, Castellón de la \\ Plana, Spain \\ ${ }^{4}$ Labpsitec, Universitat de Valencia, Valencia, Spain \\ ${ }^{5}$ Labpsitec, Universitat Jaume I, Castellón, Spain \\ *Corresponding author: mclemente@labhuman.i3bh.es
}

\begin{abstract}
In the field of virtual reality (VR), many efforts have been made to analyze presence, the sense of being in the virtual world. However, it is only recently that functional magnetic resonance imaging (fMRI) has been used to study presence during an automatic navigation through a virtual environment. In the present work, our aim was to use fMRI to study the sense of presence during a VR-free navigation task, in comparison with visualization of photographs and videos (automatic navigations through the same environment). The main goal was to analyze the usefulness of fMRI for this purpose, evaluating whether, in this context, the interaction between the subject and the environment is performed naturally, hiding the role of technology in the experience. We monitored 14 right-handed healthy females aged between 19 and 25 years. Frontal, parietal and occipital regions showed their involvement during free virtual navigation. Moreover, activation in the dorsolateral prefrontal cortex was also shown to be negatively correlated to sense of presence and the postcentral parietal cortex and insula showed a parametric increased activation according to the condition-related sense of presence, which suggests that stimulus attention and self-awareness processes related to the insula may be linked to the sense of presence.
\end{abstract}

\section{RESEARCH HIGHLIGHTS}

- We analyzed the sense of presence experienced in a virtual environment using fMRI.

- Free navigation in a virtual environment was compared with video and photographs.

- The main presence results obtained for the comparison between navigation and video.

- Cuneus, post-central parietal lobe and insula were the principal activated areas.

- Negative correlation with questionnaires found in the dorsolateral prefrontal cortex.

Keywords: presence; virtual reality; human computer interaction (HCI)

Editorial Board Member: Timothy Bickmore

Received 31 October 2012; Revised 4 April 2013; Accepted 29 June 2013

\section{INTRODUCTION}

Virtual reality (VR) is a technology that has been widely used to simulate reality. One of the most important concepts of measuring in VR applications is presence. Presence can be defined as the sense of being there, inside the virtual environment (VE), although your body is physically located 
elsewhere (Baños et al., 2000; Sadowski and Stanney, 2002; Sheridan, 1992; Slater and Wilbur, 1997). In our research, we used functional magnetic resonance imaging (fMRI) to evaluate brain activation associated with sense of presence during navigation in a VE, which, as far as we know, is a method that has not yet been applied for this purpose.

The measure of presence has a major role in the study of the human-computer interaction (HCI), in order to improve the design of VEs and to measure the effect that those improvements have over the subject. If the subject's sense of presence increases inside the VR, it means that the interaction between the computer-generated world and the subject has improved. According to Riva et al. (2003), 'as media becomes increasingly interactive, perceptually realistic and immersive, the experience of presence becomes more realistic'. Moreover, as Sjölie et al. (2010) remarked, measuring the brain activity while interacting naturally with the system allows for correlating the activity in specific brain areas with hidden cognitive states, which can be related to aspects of the interface and the interaction. The final aim in HCI is to develop systems 'that minimize the barrier between the human's cognitive model of what users want to accomplish and the computer's understanding of the users' task' (Sharp et al., 2007). If we can disclose the neural correlates that hide behind the sense of presence, maybe we will be able to develop adaptive brain-computer interfaces (BCIs) which allow the environment to change depending on the user's needs. This will help one to accomplish a satisfying experience for the user, making the VEs disappear from the subject's awareness (disappearance of mediation; Riva et al., 2003).

Although presence is inherent to exposure to VEs, most previous studies that combined VR with fMRI did not evaluate this concept or its influence on the virtual experience. The first studies in this area aimed to demonstrate the value of combining both techniques and the different possibilities that this association could offer (e.g., Baumann et al., 2003; Mraz et al., 2003). Other studies have analyzed brain activation associated with the performance of specific navigation tasks in controlled VEs. For example, Astur et al. (2005) analyzed spatial memory during navigation in a virtual radial arm maze and reported changes in bilateral hippocampus activation. Pine et al. (2002) conducted a study of the neuronal correlates of different spatial navigation conditions using VEs as stimuli. They found that navigation ability correlated with activation in different regions, including the right-frontal and rightanterior medial temporal lobe. Hartley et al. (2003) also used fMRI to compare brain activations between two experimental conditions: wayfinding and route following. They obtained between-subjects correlation of activation with performance in the anterior hippocampus for the former condition, and in the head of caudate for the latter. In another study, Mellet et al. (2010) compared brain activations while mentally estimating the distances between landmarks placed in a learned environment. One group of subjects performed the learning during a real walking task and the other in an equivalent virtual walking task of the same environment. They found a left-lateralization of the brain activations in the virtual learners, compared with the real learners. In another study, Sjölie et al. (2010) analyzed the brain activations due to a mental rotation task. Although they did not measure presence, two other important VR parameters were analyzed: 3D-motion and interactivity. For this purpose, they compared between three experimental conditions: one without motion (still), one with automatic non-interactive motion (auto) and the last one with interactively controlled motion (interactive). They found that the addition of interactivity increased the activation of the main areas of the mental rotation network, including frontal and preparatory motor areas.

Turning now to presence, several authors have theorized about the concept and its implications (e.g., Kim and Biocca, 2006; Lombard and Ditton, 2006; Schuemie et al., 2001). Inside a VE, presence refers to the sense of being in it instead of being in the real room where the experience is taking place. According to Heeter (1992), in the natural world, this process is engaged since birth; while in the VE this derives from feeling as if you existed as a separate form within a virtual world that also existed. Sanchez-Vives and Slater (2005) also pointed out that inside the virtual experience, you are at the same time conscious of the 'place' and the 'events' and simultaneously conscious of that there are no such place of events; however, you still behave and think as if the place were real and the events were happening. As your consciousness of the differences between the real and virtual place and events blurs, the barrier between your mind and the VE diminishes, improving your interaction with the computer-generated world. And that is because, as Loomis (1992) remarked, 'presence is a fundamental property of consciousness'. Therefore, it is unlikely to be unidimensional (Kim and Biocca, 2006). The International Society for Presence Research (2000) proposed that presence could be considered from several major dimensions, based on the findings of different studies in the matter. The first dimension is spatial presence, the subjects' belief that they are really inside the VE. The second is sensory presence, which is related to the subjects' perception of the VE as they would perceive the real world, divided into visual, auditory and tactile perception. Social realism refers to the subjects' perception that objects, events and people that appear in the VE could exist in the real world. Engagement occurs when the subject feels the VE to be involving. Finally, social presence refers to communication with other people or entities inside the VE.

However, many theories about what is presence have been formulated. For example, presence has been defined as the faculty of being-in-the-world (Flach and Holden, 1998; Zahorik and Jenison, 1998). In this assumption, presence is described as the 'successfully supported action in the environment'. As Sanchez-Vives and Slater (2005) explained, following this theory, presence would not only be the sense of 'being there', but also the ability to 'do' there. In this line of argument, the 
possibility of movement through the environment by the subject would increase his sense of presence.

Regarding measurement techniques for presence, traditional methods are questionnaire-based (e.g., Baños et al., 2000; Lessiter et al., 2001; Usoh et al., 2000; Witmer and Singer, 1998). To avoid the problems inherent to subjective measures, objective techniques have also been proposed, mainly based on psychophysiological measures. For example, as skin conductivity and heart rate can be related to the anxiety level of the users, they could constitute an indicator of the presence that the user is feeling in environments that generate these kinds of responses (Dillon et al., 2000; Meehan et al., 2001). Furthermore, recent studies have analyzed presence from a neuroscience point of view, and it has been stated that VR is not only a tool for neuroscience, but that presence in VEs is also an object of study for neuroscientists (Sanchez-Vives and Slater, 2005).

We can also remark some studies which focused on the concept of presence using other brain activation related techniques, such as transcranial Doppler (TCD) or electroencephalography (EEG). Two recent studies (Alcañiz et al., 2009; Rey et al., 2010) proposed that TCD could be used as a brain activity measurement technique to study presence in VEs. Results of these two studies showed changes in blood flow velocity in the major cerebral arteries of the participants during moments associated with different levels of presence in different immersive and navigational conditions. With regard to EEG, we can emphasize the work developed by Baumgartner et al. (2006), which compared activation in children and adolescents while watching a video of a virtual roller coaster and concluded that it evoked activations in parietal brain areas. Furthermore, the children reported a higher spatial presence experience than the adolescents (showed by a lower activation in the frontal brain).

However, as already indicated, there are no fMRI studies that have analyzed brain activation associated with presence during a free navigation in a VE. The advantages that fMRI provides compared to other image techniques are based on its better spatial resolution together with a decent temporal one, the fact that it is not invasive and that does not use ionizing radiation (unlike other image techniques such as computed yomography or X-rays), not provoking secondary effects on the subject. The spatial resolution of the technique is suitable to observe specific brain areas and neural networks that are activated during the presence experience, which may not be possible with other techniques. As far as we know, the only previous related study was conducted by Baumgartner et al. (2008), which applied fMRI to analyze brain activation associated with presence in VR. However, they did not use VEs in which the participants could navigate freely. They compared brain activation in children and adults while the subjects watched a VR video of an automatic navigation, with the same roller coaster scenario used in the previous EEG study (Baumgartner et al., 2006), to identify which areas of the brain were activated with the experience of presence and to identify differences between the age groups due to brain maturity. They compared the differences between a high presence and a low presence environment. Results from the fMRI analysis showed that the presence experience evoked by the virtual roller coaster scenario was associated with an increase in activation in a distributed network. A preliminary neuroanatomical model of presence can be extracted from these results. As discussed later by Jäncke et al. (2009), the distributed network that is activated during the presence experience includes the dorsal and ventral visual stream, the parietal cortex, the premotor cortex, the mesial temporal areas (including the hippocampus, amygdala and insula), the brainstem and the thalamus. Baumgartner et al. (2008) explained the activation of the parietal lobe based on the definition of presence as 'an egocentric spatial experience of VEs'. The parietal lobe is believed to be activated as a result of its role in 'generating an egocentric view by translation of the retinal coordinates to head-centered coordinates'. They also concluded that there are some brain areas, such as the prefrontal cortex, that continue maturing throughout life and may be associated with 'the modulation of the inter-individual differences in the experience of presence'. The more intense the sense of presence, the smaller was the activation in the dorsolateral prefrontal cortex (DLPFC) (Jäncke et al., 2009). They also remarked the activation of the insula as part of the distributed network that generates presence. The insula is considered to participate in the sense of self-awareness and body-ownership, which leads to the formation of your 'body schema'. This means that if this area is activated in the participants of a presence-inducing task, their feeling of embodiment inside the VE will have increased during the experience, making the barrier between the virtual and the real body disappears. This will contribute to an improvement in the participants' interaction with the VE. This concept is important when considering HCI applications of presence.

In the present study, the main goal was to analyze if brain imaging techniques can be used to evaluate the sense of presence stimulated while the subject interacts with a computergenerated environment. For this purpose, we looked for the brain areas that were activated in relation to the sense of presence during a VR paradigm. However, the most relevant objective of the present work is not only to map the brain areas related to presence, but also to study if the sense of presence itself can be stimulated and measured using fMRI. If this can be proved, it will mean that the interaction between the environment and the subject is performed naturally, and that the technology has become 'invisible' to the subject. In a previous publication, the questionnaire results of this work were presented in comparison with other brain imaging techniques (Clemente et al., 2011), now we want to complete those questionnaire results with those obtained from the fMRI data. Once the importance of the concept of presence inside the HCI theory is understood, fMRI can be seen as the tool to analyze the existence of the sense of presence in a more objective way than the use of questionnaires. What is more, fMRI allows the continuous measure of slow variations in the sense of presence in time, so it will allow us to 
have a complete report of the HCI along time; thing that cannot be accomplished with the traditional measurement tools. This can work as a steady feedback of the efficiency of the VR world.

We know from previous studies that the sense of presence is influenced by the possibility of self-controlling the navigation (Alcañiz et al., 2009; Welch et al., 1996). In order to analyze the brain activation associated to changes in the level of presence, different navigation paradigms will be compared in our experimental design. Specifically, we will compare brain activation during an experimental condition where the participants could navigate freely, with less immersive configurations (visualization of still images of the environment, and visualization of an automatic navigation - video-through the same environment). The selection of the three experimental conditions has been made based on the definition of Sanchez-Vives and Slater (2005) of the concept of presence as the ability to 'do' inside the VR, so the more you do inside the $\mathrm{VE}$, the more presence you will feel. Comparing the conditions of free and guided navigation (video), we expect to measure the differences in the level of presence due to the self-control of the movement; while the still-photographs condition will act as baseline condition. From this point of view, the increase of activity between the three experimental conditions would be translated to an increase in the sense of presence, losing the consciousness of the existing barrier between the real and the virtual world. In order to ensure that there were differences in the level of presence between the different experimental conditions, the sense of 'being there' was evaluated by means of a validated questionnaire (Usoh et al., 2000), which has been applied to obtain a subjective measure of the spatial dimension of presence in the different conditions and subjects. The main hypothesis of our research was that brain activation would be higher during a navigation task than during a video or photograph task in areas such as the cuneus and the parietal lobe, which we know are related to presence from previous studies. Taking into account the results of Baumgartner et al. (2008), we also expected to find negative correlations between the activation in the DLPFC and self-reported presence scores.

\section{MATERIALS AND METHODS}

\subsection{Subjects}

For this study, we recruited 14 right-handed women, none of them with any medical or psychological disorders, aged between 19 and 25 (mean age 21.64). The participants' hand dominance was tested using the Edinburgh Handedness Inventory (Oldfield, 1971). These women were students, were paid for their participation in the study and were recruited from the Universitat Jaume I in Castellón and the Universitat Politècnica de València.

Ethical approval was obtained from the authors' institution, and each subject signed a written informed consent prior to participation.

\subsection{Environments}

The VEs were programmed using GameStudio software (Conitec Datensysteme GmbH, Germany), which allowed us to develop 3D objects and virtual worlds with which we could interact and navigate. Our VE consisted of an everyday, clean bedroom (with a bed, a closet, and a desk with some books on it) where participants could navigate freely.

To allow us to identify the specific areas of the brain that were activated for each task, we divided the paradigm into three conditions developed with the same VE: in the first, only photographs of the room could be visualized (four photographs displayed for $4.5 \mathrm{~s}$ each with $0.5 \mathrm{~s}$ of black screen between them); in the second, a video of an automatic navigation through the same room could be observed (with a duration of $20 \mathrm{~s}$ ); and in the last one, the participant could navigate freely for $20 \mathrm{~s}$ in the VE.

In order to prevent the subjects from staying still during the navigation period, they were instructed to perform a search task which forced them to move through the environment and kept them engaged with the stimuli. This task consisted in searching for some red keys that randomly appeared and disappeared in the environment, and counting the number of them that they had seen (maximum of 4 , remaining in the VE for $5 \mathrm{~s}$ ). They were not encouraged to find them all, or to find them as quickly as possible, they were only told to continue searching for them during each period. To prevent differences between the different phases of the experiment, this counting task was also performed during the other two conditions. During the photograph period, some of the images showed featured keys and some did not, and the subjects had to count the number of keys they saw. During the video task, the keys appeared randomly in the environment as the camera moved through it. After each task, subjects were questioned about the number of keys they had found (they had to answer in a short period of $4 \mathrm{~s}$ ). While they were conducting the tasks, the researcher checked that they had answered properly. The number of keys counted is not relevant, it was just included to avoid the subjects to remain still during the experimental conditions. Between phases, a black screen appeared to give subjects a rest period during which brain activation could decay to its baseline values $(6 \mathrm{~s})$ before the label indicating the next task appeared ( $2 \mathrm{~s})$. The total time between tasks was $12 \mathrm{~s}$. At the beginning of the experiment there were $14 \mathrm{~s}$ of black screen to compensate for T1 saturation effects. Each of the three experimental conditions was repeated six times in a counterbalanced order to prevent effects produced by the order in which they were presented. The total time of the complete experiment was $12 \mathrm{~min} 52 \mathrm{~s}$. A scheme of the protocol can be seen in Figure 1.

To learn about the tasks that had to be performed inside the scanner room, subjects underwent a prior training session where they were introduced to the VR navigation and to the tasks. They were also shown the differences between the photographs, videos and navigation, and practiced the hand movement using the joystick as it was going to be done during the scanner session. 

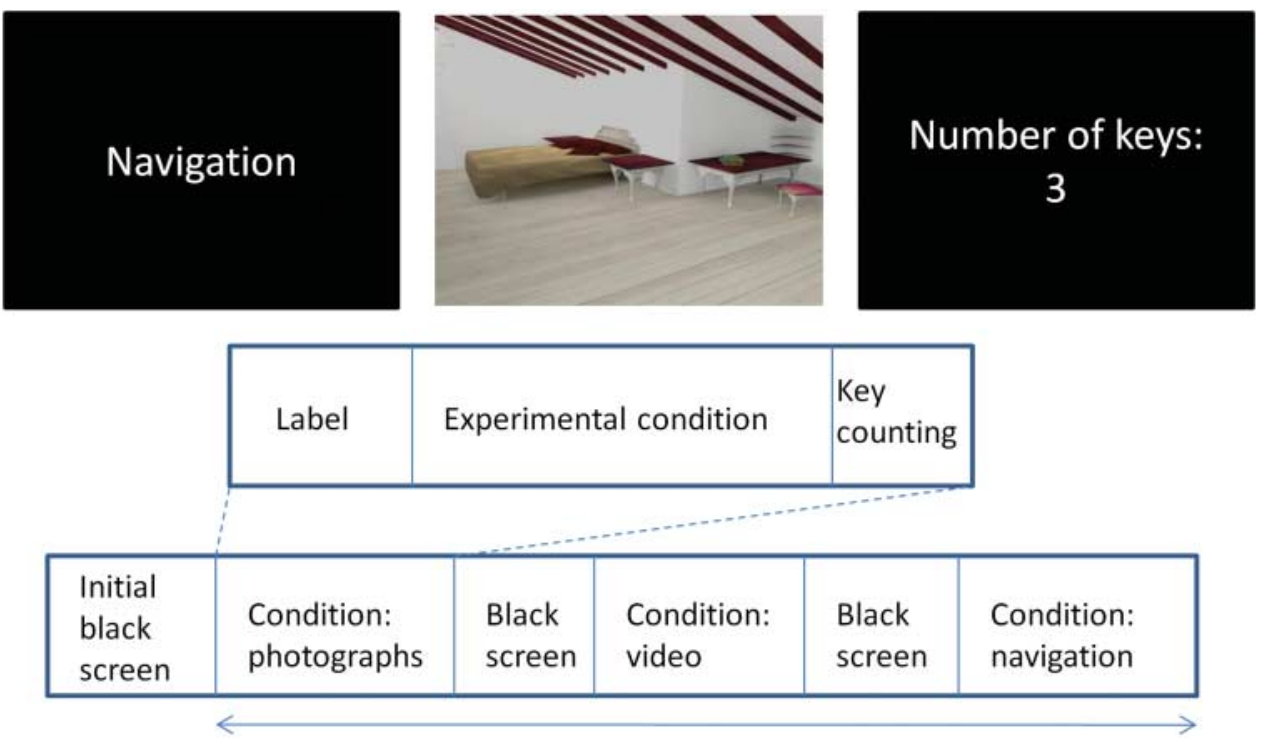

Repeated in a counterbalanced order

FIGURE 1. Diagram of the experimental design.

This training session was conducted in a supplementary VE to prevent habituation. In order to prevent differences in activation caused by the motor task, subjects were instructed to move the joystick continuously during the video and photograph tasks in the same way as they did during the navigation period. The joystick movement was made just to compensate the brain activations caused by motor tasks between the different conditions. During the fMRI scan, the VR application checked the total time that they spent moving the joystick in each condition to guarantee that the motor movements had been continuous in all the cases.

\subsection{Post-fMRI questionnaires}

After the scanner session, subjects had to answer the questions in the Slater, Usoh and Steed (SUS) questionnaire (Usoh et al., 2000) to evaluate the level of presence that they felt during each task. The questionnaire consisted in six, 7-point Likert-type, questions that had to be answered depending on the strength of the 'being there' sensation experienced, where 1 corresponded to not feeling there at all and 7 to the highest sense of being there (as experienced in the real world). A midscale value of 4 would correspond to an intermediate level of being there, experienced by the subject as the midpoint between the feeling in the real world and not feeling there at all. Subjects had to complete three questionnaires, one for each task, all containing the same questions.

\section{4. fMRI procedures}

All subjects were scanned in a 1.5 T Siemens Avanto Magnetic Resonance scanning device (General Hospital, Castellón,
Spain). We used an adapted magnetic resonance (MR) helmet to prevent head movement. To display the environments, we used MRI-compatible video goggles, VisualStim Digital (Resonance Technology Inc., Los Angeles, USA); and, for the navigation, we used an adapted joystick (Resonance Technology Inc., Los Angeles, USA). First, as is indicated for fMRI studies (Amaro and Barker, 2006), we acquired the sagittal T1-weighted structural images of the brain $(224 \times 256$ matrix covering the brain with 176 contiguous $1 \mathrm{~mm}$ slices, repetition time $(\mathrm{TR})=$ $11 \mathrm{~ms}$, echo time $(\mathrm{TE})=4.94 \mathrm{~ms}$, flip angle $(\mathrm{FA})=15^{\circ}$, voxel size $=1.04 \times 1.04 \mathrm{~mm})$. Then the functional scanning was launched, synchronized with the VEs. Functional images were obtained using a T2* single-shot echo-planar imaging sequence. We used 30 contiguous $4.2 \mathrm{~mm}$ interleaved axial slices (parallel to the line between the anterior commissure $(\mathrm{AC})$ and the posterior commissure (PC) or the AC-PC line) covering the entire volume of the brain with a $64 \times 64$ matrix $(\mathrm{TR}=2000 \mathrm{~ms}$, $\mathrm{TE}=30 \mathrm{~ms}, \mathrm{FA}=90^{\circ}$, voxel size $\left.=3.5 \times 3.5 \mathrm{~mm}\right)$.

\subsection{Data analysis}

\subsubsection{Questionnaire analysis}

We analyzed the results of the SUS questionnaires using the program SPSS 17.0 (IBM Corporation, Somers, NY, USA). Apart from the individual responses to the six questions associated with each of the periods (photographs, video and navigation), we calculated an additional measurement: SUS mean. This is the mean score across the six questions that have already been described in previous studies (Usoh et al., 2000). We carried out a non-parametric Friedman Test to compare SUS responses (dependent variables: questions 1-6 and the SUS 
mean) for the different experimental conditions: photographs, video and navigation. Post hoc tests were made with a Wilcoxon Signed-Rank test with Bonferroni correction.

\subsection{2. fMRI analysis}

To analyze the fMRI data we used Statistical Parametric Mapping software (SPM8, Wellcome Department of Imaging Neuroscience, London, UK), launched with Matlab Version 7.1 (MathWorks, Natick, MA, USA). The first seven scans were excluded from the analysis to eliminate the decay of the fMRI signal associated with the moment when magnetization reaches equilibrium. The first step was to align the images to the ACPC line. Then we began the preprocess (Friston et al., 1995) realigning the functional images (estimate and reslice option), coregistering them to the structural images and segmenting this latter anatomical scan to obtain the gray matter, white matter and cerebrospinal fluid images. We then normalized the resliced functional volumes with the normalization parameters extracted after segmentation and normalization of the anatomical volumes for each subject separately (template provided by the Montreal Neurological Institute [MNI]). We did not have to exclude any volunteer due to movements or distortions during the fMRI. Finally, we smoothed the images using a Gaussian kernel (fullwidth at half-maximum of $8 \times 8 \times 8 \mathrm{~mm}$ ).

In a first fixed-effect level analysis (with the aim of detecting changes in the blood-oxygen-level-dependent (BOLD) signal between conditions in a single subject), the individual contrasts comparing between the different experimental conditions are obtained. In this analysis, the functional time series for each subject and for each condition were modeled with a box-car function convolved with the hemodynamic response function. As a result, the 'navigation > video', 'navigation > photographs' and 'video > photographs' contrasts for each subject were obtained. The parameters for the motion correction were employed as regressors of non-interest. We also applied a $128 \mathrm{~s}$ high-pass filter to eliminate the lowfrequency components in the signal caused by scanner motion and warming.

Group tests were performed at second level random effect analysis, where the group of subjects is taken into account. Taking into account the results obtained in previous similar studies (Baumgartner et al., 2008; Pine et al., 2002), we tested for task-related activation by performing a one-sample $t$-test including contrast images of estimated parameters from all the subjects for the differences of interest between conditions. In total, we performed three one-sample $t$-test, for the contrasts 'navigation > video', 'navigation > photographs' and 'video > photographs'.

Once we obtained the brain activation maps for each grouplevel contrast, we conducted a second-level multiple regression analysis to evaluate the existent relationship between brain activation in the aforementioned contrasts ('navigation > video', 'navigation > photographs' and 'video > photographs') and the subjective scores from the questionnaires. We performed three new group-level analysis (for the three contrasts of interest), where we used as covariate the differences between the SUS mean results for the experimental conditions compared in the contrast (see Baumgartner et al., 2008). The covariate then is a vector of 14 components, one for each subject. The value of the component of each subject is obtained by subtracting the value of the SUS mean for the second condition of the contrast compared from the value of the SUS mean of the first condition of the contrast. For example, our main interest lay in the 'navigation > video' contrast, where we obtained the correlation analysis between the 'navigation > video' contrast and the responses from the SUS questionnaires for the 'navigation SUS mean score-video SUS mean score'.

Finally, we studied the brain areas that showed a linear parametric modulation of the activation levels and their associated subjective level of presence (SUS mean of each condition minus the global SUS mean) for the three experimental conditions (navigation, videos and photographs), according to increased sense of presence (following a procedure described in previous studies, such as Geake and Hansen, 2005; Scheibe et al., 2006; Smith, 2004).

Results from statistical tests at group level were considered significant if 10 or more adjacent voxels passed the statistical threshold of $p<0.001$ (uncorrected). To obtain the specific brain areas that are activated in each contrast, we used the xjView (http://www.alivelearn.net/xjview8/) software utility for SPM that uses the MNI coordinates system.

\section{RESULTS}

\subsection{Questionnaire results}

The answers to the SUS questionnaire, given after fMRI monitoring during exposure to the different experimental conditions, showed the between-subject variations. As sense of presence is subjective, each person can experience the conditions with a different grade of affectation. Mean values in each condition are shown in Table 1.

Results from applying the non-parametric Friedman Test showed that there were significant differences between the three experimental conditions for all the questions except question 5 (results can be observed in Table 1, columns 5 and 6), including the SUS mean value. If we observe the results for each question, we can see that the greatest Chi-square value $\left(\chi^{2}=16\right.$, $p<0.001$ ) is observed for question 1 .

Post hoc analyses based on Wilcoxon Signed-Rank tests were conducted on the SUS mean results with Bonferroni correction, resulting in a significance level set at $p<0.0167$. There were no significant differences between the photograph and the video tasks $(Z=1.174, p=0.241>0.0167)$. However, there was a statistically significant increment in the SUS mean in the navigation versus photographs $(Z=2.805, p=0.005<$ $0.0167)$ and the navigation versus video comparisons $(Z=$ $2.550, p=0.011<0.0167)$. 
TABLE 1. SUS responses to the questionnaires for each task and results of the Friedman test for each question (7-point Likert scale) and the mean score

\begin{tabular}{|c|c|c|c|c|c|}
\hline & Photographs & Video & Navigation & $\chi^{2}$ & $\mathrm{P}$ \\
\hline SUS question 1: feeling of 'being there' & $3.14 \pm 0.39$ & $3.79 \pm 0.45$ & $4.43 \pm 0.43$ & 16.00 & $<0.001$ \\
\hline SUS question 2: feeling that the room is real & $2.79 \pm 0.48$ & $3.14 \pm 0.48$ & $3.50 \pm 0.50$ & 6.75 & 0.034 \\
\hline SUS question 3: how real do you remember the room? & $2.00 \pm 0.31$ & $2.50 \pm 0.42$ & $3.14 \pm 0.49$ & 10.90 & 0.004 \\
\hline SUS question 4: feeling of being inside the room or observing it & $3.14 \pm 0.38$ & $3.14 \pm 0.49$ & $4.07 \pm 0.45$ & 6.45 & 0.004 \\
\hline SUS question 5: memory of the room as similar to being in other places & $3.43 \pm 0.44$ & $3.50 \pm 0.42$ & $4.00 \pm 0.46$ & 5.25 & 0.072 \\
\hline SUS question 6: did you think you were really in the room? & $2.71 \pm 0.37$ & $3.00 \pm 0.50$ & $3.50 \pm 0.53$ & 6.07 & 0.048 \\
\hline SUS mean & $2.87 \pm 0.33$ & $3.18 \pm 0.40$ & $3.77 \pm 0.43$ & 12.29 & 0.002 \\
\hline
\end{tabular}

Note. The mean score and standard error of the mean for the 14 subjects are presented in each cell.

TABLE 2. Brain area activation results for the 'navigation $>$ video', 'navigation $>$ photographs' and 'video $>$ photographs' contrasts

\begin{tabular}{|c|c|c|c|c|c|c|c|}
\hline & Anatomical region & $\mathrm{H}$ & $x(\mathrm{~mm})$ & $y(\mathrm{~mm})$ & $z(\mathrm{~mm})$ & $t$ score & Cluster size \\
\hline \multirow{5}{*}{ Navigation $>$ Video } & Postcentral/Parietal Lobe & $\mathrm{L}$ & -47 & -18 & 59 & 5.7771 & 19 \\
\hline & Insula & $\mathrm{R}$ & 34 & 0 & 17 & 5.4986 & 10 \\
\hline & Cuneus/Occipital Lobe & $\mathrm{R}$ & 10 & -91 & 26 & 5.3162 & 10 \\
\hline & Calcarine/Middle Occipital gyrus/Occipital Lobe & $\mathrm{R}$ & 24 & -98 & 0 & 5.0042 & 22 \\
\hline & Cerebellum Anterior Lobe & $\mathrm{L}$ & -43 & -49 & -37 & 5.0356 & 56 \\
\hline Navigation $>$ Photographs & Lingual/Occipital Lobe & $\mathrm{R}$ & 3 & -70 & 5 & 6.7976 & 83 \\
\hline \multirow[b]{4}{*}{ Video $>$ Photographs } & Superior Frontal Lobe & $\mathrm{R}$ & 24 & 0 & 55 & 9.8225 & 143 \\
\hline & Inferior Temporal Lobe/BA37 & $\mathrm{R}$ & 48 & -70 & -4 & 9.57 & 1147 \\
\hline & Lingual Gyrus/Inter-Hemispheric & $\mathrm{R}$ & 3 & -74 & 0 & 5.28 & 38 \\
\hline & Inferior Frontal Operculum/Sub-Gyral/Frontal Lobe & $\mathrm{R}$ & 41 & 11 & 21 & 5.99 & 44 \\
\hline
\end{tabular}

Note: $\mathrm{H}$ column refers to the hemisphere of each activation.

\subsection{Imaging results}

\subsubsection{Contrasts results}

Our fMRI paradigm was divided into three different tasks (photographs, videos and navigation) that we wanted to compare to obtain the contrasting brain activations. We obtained results for the three contrasts between tasks, the most relevant for the purposes of our study being those concerning the differences in activation between the free navigation and the guided navigation (video). Therefore, we selected the contrast 'navigation > video' and looked for the main activated brain regions. We found activations in the right cuneus and left parietal lobe among others (see the upper part of Table 2 and Figure 2). Other brain regions activated in the 'navigation $>$ video' contrast were the right calcarine, right sub-lobar and right insula.

Regarding the 'navigation > photographs' contrast, new activations were seen in the left cerebellum, both in the anterior and posterior lobes, and in the superior frontal lobe. There were activations in some areas of the occipital lobe, such as the cuneus, the left and right middle occipital lobe, and the right lingual gyrus. Finally, we also found activations in areas of the parietal lobe, such as the precuneus (see the middle part of Table 2 and Figure 3).

For the 'video $>$ photographs' contrast, we found activations in the right inferior temporal lobe, the right lingual gyrus, the right inferior frontal lobe, the right supramarginal gyrus and the right and left middle frontal lobe (see the inferior part of Table 2 and Figure 4). It is important to mention here that with the inverse contrasts ('video $>$ navigation', 'photographs > navigation' and 'photographs $>$ video'), we did not obtain any significant activation results.

\subsubsection{Positive and negative correlation results}

We conducted a second-level multiple regression analysis for our contrast of interest 'navigation > video', from which we obtained correlations between the fMRI results and the SUS mean values obtained from the questionnaires. On the one hand, for the 'navigation > video' contrast, results showed a negative correlation (Table 3 ) between the questionnaire results 

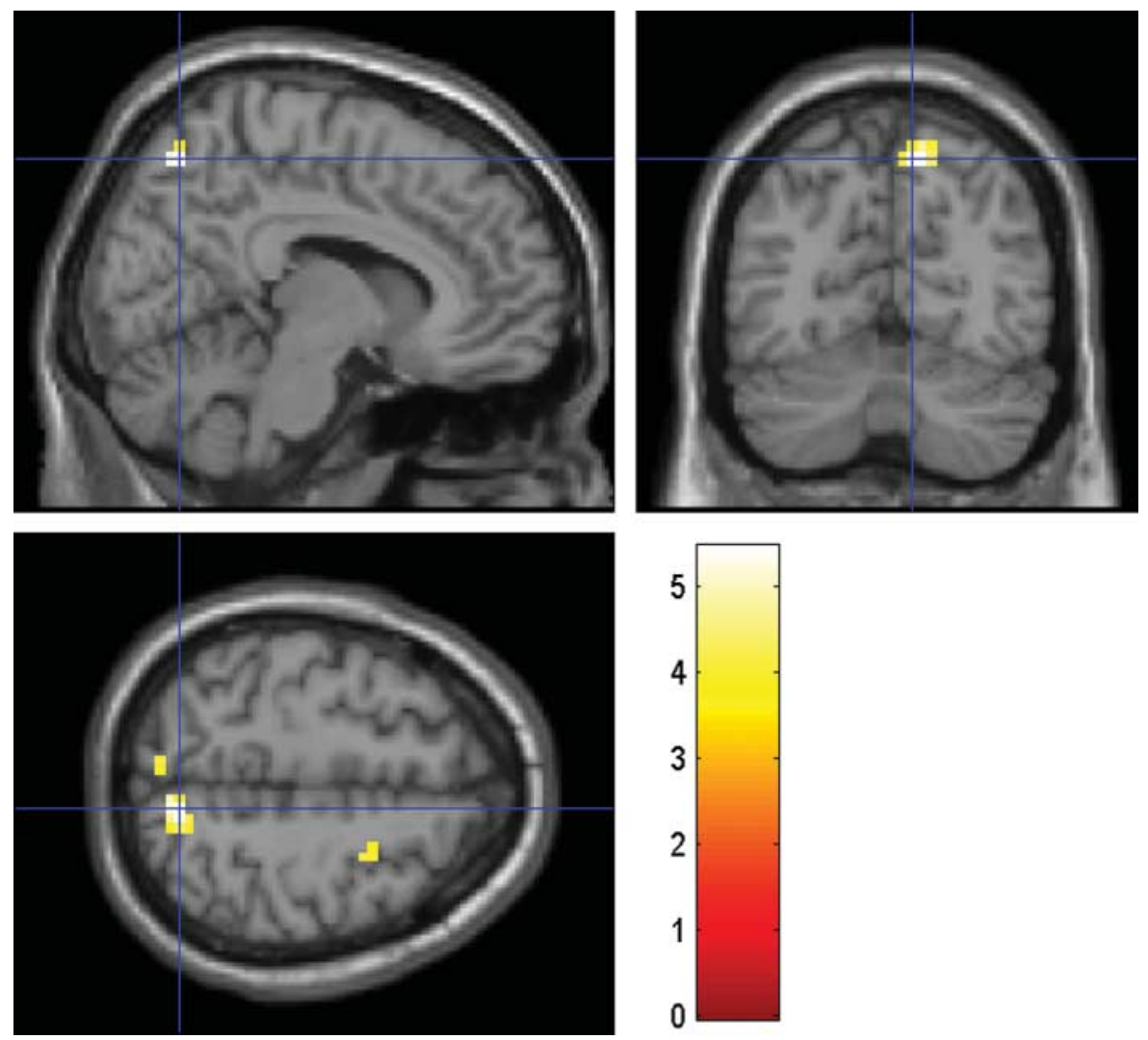

FIGURE 2. Main brain activation results for the 'navigation $>$ video' contrast, centered in the right cuneus. Results are shown in neurological coordinates. The bar represents statistical $t$-values.

and the activation observed in the DLPFC of the right frontal lobe, which can be seen in Figure 5. On the other hand, a positive correlation was found between the responses to the questionnaires and activation in the left lingual gyrus, left cerebellum anterior lobe, left middle inferior temporal lobe, left sub-gyral, left calcarine, left superior temporal lobe, left middle temporal gyrus and left cuneus. All these results can be seen in Table 3. Moreover, the graph showing the positive correlation for the lingual gyrus (the most remarkable of the positive correlation results) is shown in Figure 6.

\subsubsection{Parametric analysis results}

Finally, we tested for the possible existence of an increasing linear trend in the activation corresponding to the three reported levels of presence (SUS mean of each condition minus the global SUS mean values) according to the experimental conditions. The results showed that an increasing linear trend for the different presence-related conditions (photographs, videos and navigation) was observed in the activations in the right insula $(x=41, y=-14, z=13 ; t=4.22, p<0.001,10$ cluster size) and the left postcentral parietal gyrus $(x=-47, y=-18$, $z=59, t=6.67, p<0.001,10$ cluster size) for the three experimental conditions (see Figure 7).

\section{DISCUSSION}

The principal aim of our study was to analyze whether subjects could feel presence while navigating in a VE, analyzing the results using fMRI. As mentioned in Section 1, if this is so, this would mean that the interaction between the computergenerated world and the subject is naturally performed and the barrier between technology and reality has been reduced; which is a major interest in HCI. As mentioned previously, we tried to generate an increase in the sense of presence between the different experimental conditions by means of the increase in the actions the user has to perform in the VE. Presence was then especially motivated by the free navigation condition, where it is the user who controls the movement along the environment. This free navigation in a VE was shown to induce a higher feeling of presence in the participants than a guided navigation condition (that in a comparison would act as the low presence condition). Contrasting the functional activation seen during these two conditions ('navigation > video'), results showed a higher activation of the parietal and occipital brain regions, including the cuneus, during the navigation condition, as hypothesized, but also activation of the right insula. These areas are included in the distributed network activated by presence that was described in Section 1 of this paper. 

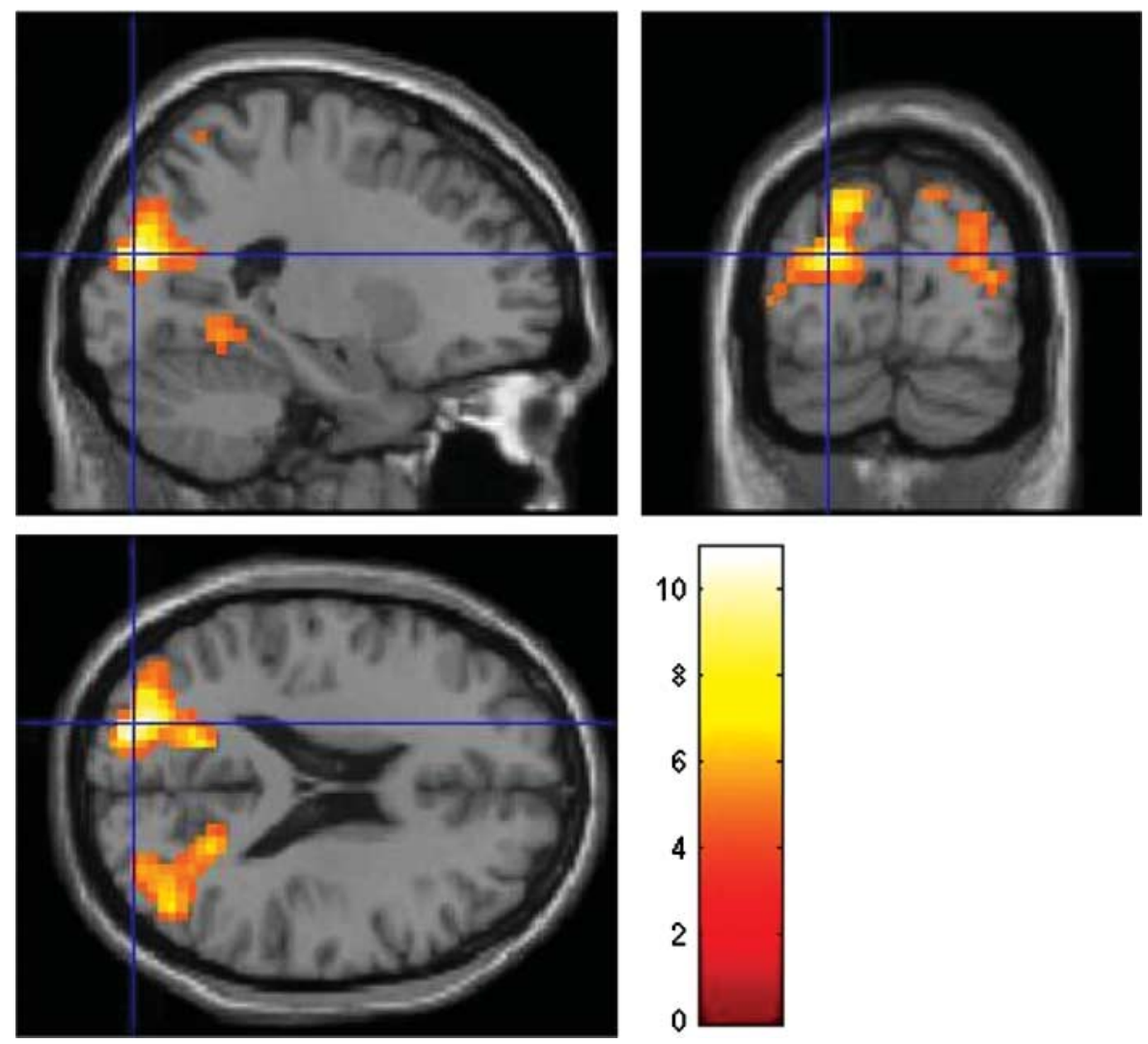

FIGURE 3. Main brain activation results for the 'navigation $>$ photographs' contrast, centered in the left superior occipital lobe. Results are shown in neurological coordinates. The bar represents statistical $t$-values.

Moreover, the differential subjective sense of presence reported by participants in the questionnaires between the navigation and video conditions was shown to be inversely correlated to the activation of the DLPFC, and directly correlated to the activation of the lingual gyrus and cuneus and other occipital and temporal regions. Finally, we observed a linear increase in the activation of the right insula and left postcentral parietal regions according to the subjective sense of presence reported for each condition (SUS mean of each condition minus the global SUS mean values). Among the multiple brain areas activated by the 'navigation > video' contrast, we can highlight the cuneus and the post-central parietal lobe, which have been related to working memory and navigation tasks (Haldane et al., 2008; Mishkin and Ungerleider, 1982). These results are comparable to those obtained in other presence studies that have been conducted using fMRI (Baumgartner et al., 2008) or can be extrapolated to the results obtained with other techniques such as TCD (Alcañiz et al., 2009) or EEG (Baumgartner et al., 2006), always considering the limited spatial resolution of these techniques. Regarding the 'navigation $>$ photographs' and 'video > photographs' contrasts, they showed some similar activations, such as the lingual gyrus, the cuneus, the frontal lobe or the occipital lobe. In this section, we will discuss all these items in more detail.
The subjects answered three SUS questionnaires (one for each experimental condition) where they evaluated the level of presence they felt. In each question, they value between 1 (not feeling there at all) and 7 (highest sense of 'being there') the presence experience. The results confirmed that a higher level of presence was induced during the free navigation than during the photograph and guided navigation conditions. Furthermore, we can observe how the mean value for subjective sense of presence increased for each condition, observing the lowest score for the photographs and the highest score for navigation. Specifically, the Friedman Test showed significant differences between the experimental conditions for all the questions and the SUS mean except for question 5, which evaluated how the user remembered the experience in comparison to a real one. The largest difference between experimental conditions in response to the questionnaire was found in Question 1, which asked directly about the sense of being in the virtual world. Finally, post hoc analysis based on the Wilcoxon Signed-Rank tests showed no significant differences in the comparison of the photograph and the video tasks, but that there were significant differences for the other two comparisons: photographs versus navigation and video versus navigation. Therefore, as hypothesized, there were significant differences between the level of presence experienced during the navigation 

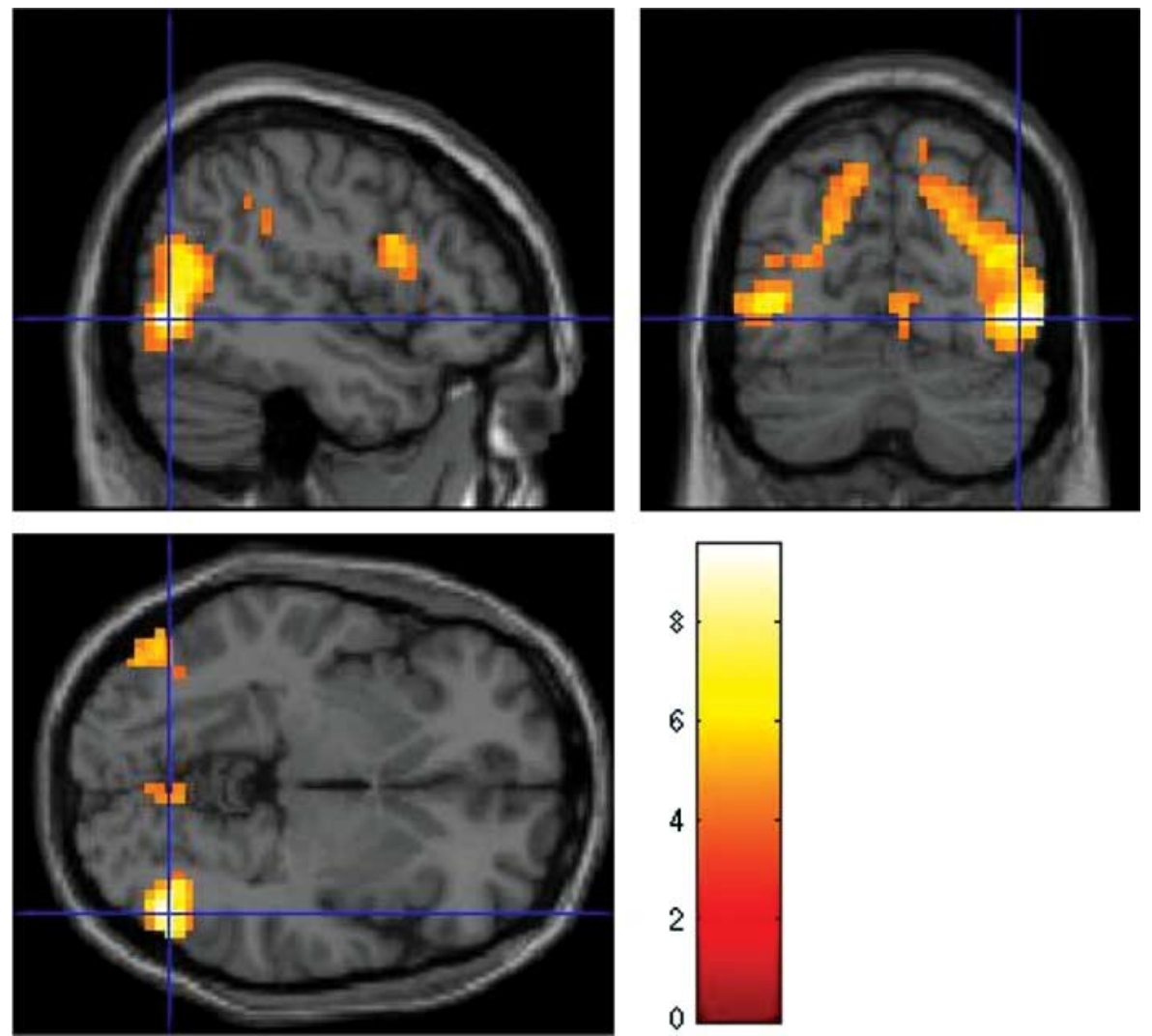

FIGURE 4. Main brain activation results for the 'video $>$ photographs' contrast, centered in the right inferior temporal lobe. Results are shown in neurological coordinates. The bar represents statistical $t$-values.

TABLE 3. Brain areas showing negative and positive correlations between activation in the 'navigation $>$ video' contrast and averaged questionnaire results.

\begin{tabular}{|c|c|c|c|c|c|c|c|}
\hline Anatomical region & Hemisphere & $x(\mathrm{~mm})$ & $y(\mathrm{~mm})$ & $z(\mathrm{~mm})$ & $t$ score & Cluster size & Correlation \\
\hline Frontal Lobe & $\mathrm{R}$ & 45 & 21 & 17 & 4.8747 & 21 & Negative \\
\hline Lingual/Parahippocampal Gyrus/Limbic Lobe & $\mathrm{L}$ & -19 & -53 & 0 & 6.1862 & 114 & Positive \\
\hline Temporal_Mid/Inferior Temporal Gyrus/Temporal Lobe & $\mathrm{L}$ & -61 & -14 & -21 & 5.9193 & 25 & Positive \\
\hline Cerebellum_4_5/Culmen/Cerebellum Anterior Lobe & $\mathrm{L}$ & -19 & -35 & -21 & 5.763 & 18 & Positive \\
\hline Cuneus/Precuneus/Parietal Lobe & $\mathrm{L}$ & -12 & -74 & 38 & 5.7275 & 23 & Positive \\
\hline Temporal_Sup/Superior Temporal Gyrus/Temporal Lobe & $\mathrm{L}$ & -68 & -28 & 9 & 5.3975 & 21 & Positive \\
\hline Sub-Gyral/Temporal Lobe & $\mathrm{L}$ & -36 & -11 & -12 & 5.1038 & 14 & Positive \\
\hline Calcarine/Cuneus/Occipital Lobe & $\mathrm{L}$ & -1 & -91 & 9 & 5.044 & 13 & Positive \\
\hline Middle Temporal Gyrus/Temporal Lobe & $\mathrm{L}$ & -29 & -74 & 17 & 4.6807 & 10 & Positive \\
\hline
\end{tabular}

condition and that experienced during the other two conditions. As indicated, a previous study by Welch et al. (1996) analyzed this connection between presence and navigation and their results are in accordance with the present study. They used two levels of interaction, the subject as an active or a passive driver, and observed that the interactivity increased the sense of presence the subject experienced.

One purpose of our research was to test the hypothesis that fMRI is an appropriate way to explore brain activation related to presence in a VE when comparing between different experimental conditions, allowing us to obtain objective differences in brain activation associated with the different levels of presence that the subjects have experienced. The main contrast that we analyzed was the 'navigation > video' contrast, to evaluate the differences in brain activation between two conditions that induced different levels of presence, as measured with the SUS questionnaire. In the following paragraphs, the results from this principal contrast will be analyzed in detail. As explained in Section 3, one of the most significant activated areas is the cuneus, part of the occipital lobe. This area has been 

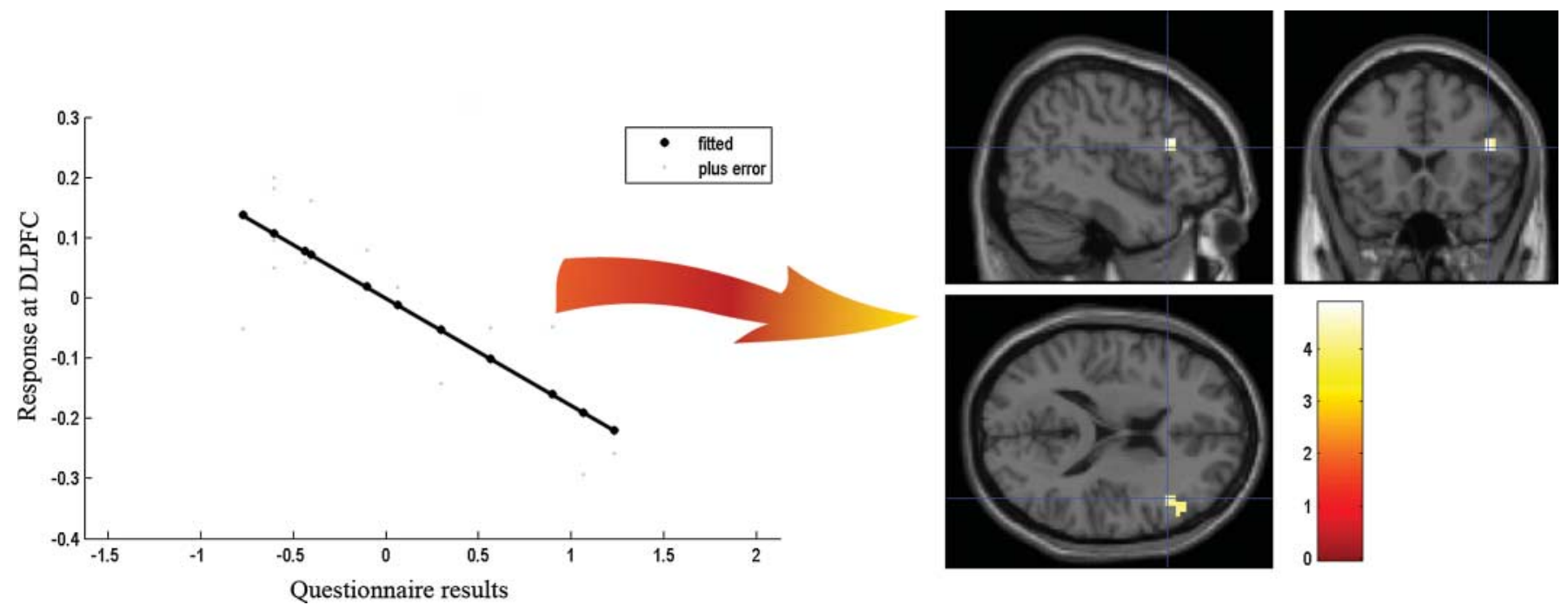

FIGURE 5. Graph of results for the 'navigation > video' contrast, showing the negative correlation between the activation in the DLPFC (contrast estimates difference) and the questionnaire results (navigation SUS mean - video SUS mean). The bar represents statistical $t$-values.
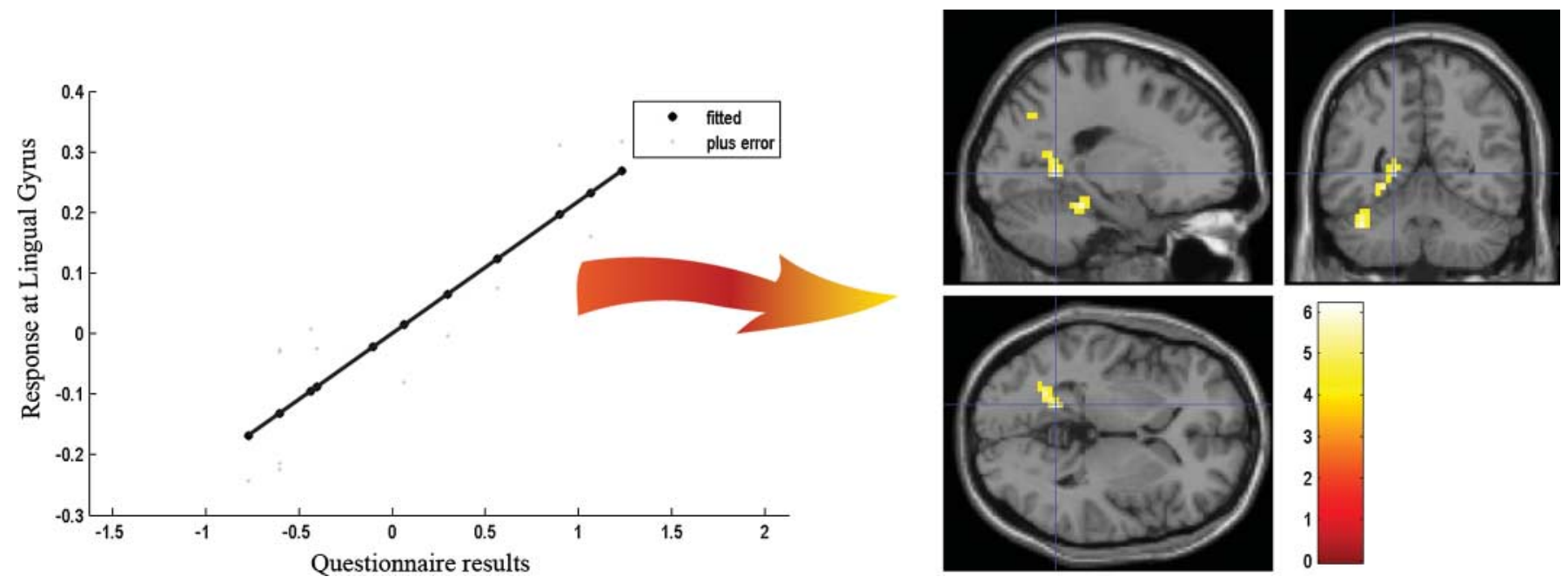

FIGURE 6. Graph of results for the 'navigation > video' contrast, showing the positive correlation between the activation in the lingual gyrus (contrast estimates difference) and the questionnaire results (navigation SUS mean - video SUS mean). The bar represents statistical $t$-values.

related in previous studies to the visual processing (Perani et al., 2001). The cuneus is known to receive visual information from the contralateral superior retina, and the processing that occurs in the area is modulated by other effects, such as attention, working memory or reward expectation (Haldane et al., 2008; Vanni et al., 2001). Our study relates cuneus activation to the subjective sense of presence experienced during free navigation in a VE. Another region included in the results is the calcarine sulcus, also part of the occipital lobe where the primary visual cortex is concentrated (Belliveau et al., 1991; Le Bihan et al., 1993).

Another brain region that showed significant activation during the task was the post-central parietal lobe. Between the usual areas considered to be part of the presence network, the parietal lobe is involved in determining spatial sense and navigation, directly associated with the navigation in the VE (Johnson et al., 1996; Mishkin and Ungerleider, 1982). Moreover, Mellet et al. (2010) found that left activation of the parietal lobe was higher while navigating through a VE than while navigating through a real one. We also found activation in the insula, usually related to emotion and the regulation of the body's homeostasis, including perception, motor control of hand and eye movements, self-awareness, cognitive functioning and interpersonal experience (Craig, 2009; Karnath et al., 2005). As pointed out in Section 1, the most important of these items for our study are self-awareness, sense of agency and sense 


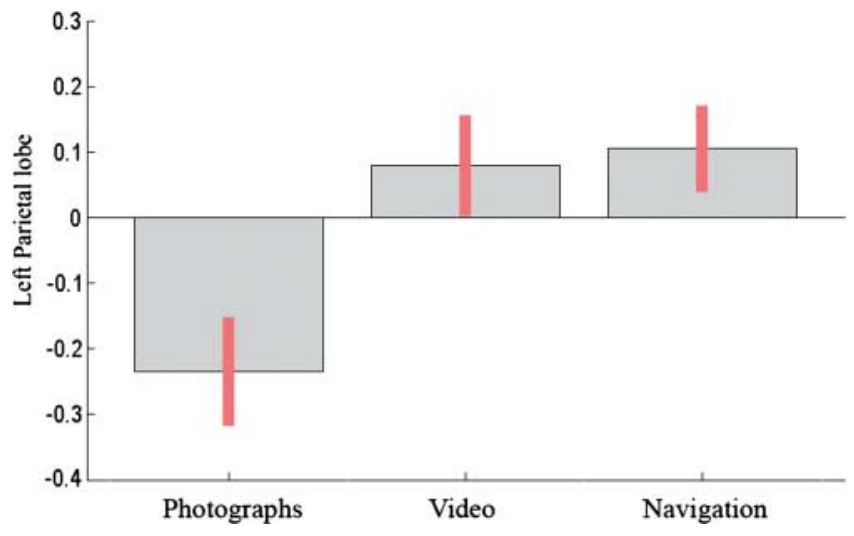

FIGURE 7. BOLD signal change in response to the different experimental conditions around MNI coordinates $(-46,-18,59)$, corresponding to the left parietal lobe. Observe the increase in signal between experimental conditions.

of body ownership, because they are closely related to the sense of presence experienced inside the VE. The sense of body ownership allows you to discriminate your individual's own body and perceptions; forming the 'body schema' that covers the dynamic distributed network of procedures aimed at guiding your behavior (Haans and Ijsselsteijn, 2012). Our results showed a parametric increase in the activation of the right insula according to the sense of presence experience in the conditions. Recent studies (Dodds et al., 2011) have found evidence that the right insula may be activated by a combination of attentional and response control demands, playing a role in the processing of sensory stimuli that are relevant to current goals. During navigation in a virtual world, decisions are constantly made based on evaluation of the sensory stimuli guiding our behavior in the VE. Our results suggest that the insula may play a key role in guiding behavior in the VE based on the presented stimuli and the sense of presence. Moreover, according to Sjölie (2012), attention and behavior are essential to develop the sense of presence, increasing the precision in the predictions about the environment and the synchronization with it, and avoiding prediction errors from sources outside the VE.

All these results are consistent with those obtained in the Baumgartner et al. (2008) research. They generated different levels of presence by means of two different types of environment, one that induced a high arousal experience and another that induced a low arousal experience. They placed particular emphasis on the parietal lobe as one of the most important areas related to presence and egocentric spatial processing, something which was also observed in our results. They also mentioned significant activations in the cuneus, middle occipital gyrus and areas involved in emotional processing, such as the insula; activations in these brain regions were also observed in our study and associated with the condition which induced the higher level of presence.
Although our results can be compared with those obtained in previous presence studies, those comparisons should be done carefully. Each brain area is involved in several other functions not related with presence, and the network described before is not a closed one to the study of presence. The activation of those areas does not necessarily imply stimulation of the sense of presence. As Jäncke et al. (2009) explained, it is a network involved in the control of many other psychological functions, and 'the psychological specificity cannot be inferred simply by identifying the activated brain structures'. Moreover, our primary aim was to demonstrate the validity of fMRI as a tool to evaluate presence; not to map the brain network involved in its stimulation. The fMRI is a great tool to measure brain activity, but the size and characteristics of the machine makes impossible to use it in real situations. If it can be proved that inside the fMRI, the subject can feel presence inside a VE; this could lead to the use of VR to approach the subject to the equivalent real situation while being scanned. Moreover, as aforementioned, demonstrating that the sense of presence can be stimulated proves that the interaction between the computer-generated environment and the subject is performed naturally, making the technology 'invisible' to the user. Obtaining activation in brain areas that have been previously related to presence is remarkable in the sense of showing that our results are not random, and that our initial hypothesis has been accomplished. The main objective of our fMRI research is then to bring into agreement with previous presence theories, not to show new results on the matter.

In a more theoretical perspective, the degree of presence in a VE may be considered as the degree of synchronization between the environment and the subject's mental reality. In our case, the subjects view the VE for the first time in their lives during the scan, but due to the increasing familiarity of humans with virtual phenomena, this should lead to the internalization of mental simulations of the VEs, which matches with the activity theory so popular in the HCI circles (Sjölie, 2012). So, the fact that the subjects are not familiarized with the environments should not prevent the sense of presence. Moreover, the central nervous system is capable of incorporating the new tools and technological artifacts that we use in the virtual experiences to its representation of the body schema, integrating them in a functional unity with our biological limbs and sensory receptors (Haans and Ijsselsteijn, 2012), helping the interface transparency or 'disappearance of mediation' (Riva et al., 2003).

Referring to the search task the subjects had to perform inside the environment, it was designed to avoid them staying still during the experiences, but the fact of identifying an objective to perform inside the virtual world enhances a major sense of presence in the subjects (Riva et al., 2011). In fact, if the performer becomes 'emotionally and intellectually engaged' by the task developed, higher levels of presence can be achieved (Waterworth et al., 2002); which leads to a state of loss of self-consciousness (Riva et al., 2011), as we previously discussed. 
We will now discuss the other contrasts evaluated in our study. As we have previously said, the results for the 'navigation $>$ photographs' contrast showed the activation of the cuneus and the parietal lobe, two of the most important results obtained in the 'navigation > video' contrast, which we have highlighted as having been previously related to presence during the navigation task. Moreover, the activation of the cuneus may reflect an increase in the visual processing due to the change in the optical flow between both conditions. We have also found activations in the cerebellum and the frontal lobe, results that coincide with those obtained by Pine et al. (2002), who also evaluated differences between free and guided navigation. The cerebellum may have been activated because of its role in the control of movement (Willshaw, 1999; Wolf et al., 2009). The frontal lobe is related to the planning of the navigation task (Baumgartner et al., 2006; Owen et al., 1990). It is also important to remark that the cuneus, precuneus, middle occipital lobe and frontal lobes are areas which were also activated in the research by Baumgartner et al. (2008).

Regarding the 'video $>$ photographs' results, there are coincidences with the study by Pine et al. (2002) in the temporal and frontal lobes. Our results agree only with those from Baumgartner et al. (2008) in the middle frontal lobe, which is often referred to as being involved in various executive functions as, for example, the planning of movement (Baumgartner et al., 2006; Owen et al., 1990). The fact that there are no other coincidences between the results may be explained by the lower sense of presence stimulated during the two conditions (video and photographs) compared here. Moreover, inside the temporo-occipital cluster, it is worthy to remark the bilateral activation of the V5/MT area, part of the extrastriate visual cortex, which plays a main role in the perception of movement (Born and Bradley, 2005), due to the addition of visual movement in the video condition.

With regard to the correlation analysis comparing brain activation and responses to questionnaires, we found a negative correlation in the prefrontal cortex, more specifically in the dorsolateral area, which agrees with the result obtained by Baumgartner et al. (2008) for the measurement of presence in video tasks, although at an inferior location within the DLPFC. This area is related to executive processing within working memory (Petrides, 2000) and controls the visual information that comes from the visualization of the $\mathrm{VE}$, being involved in the decrease of the sense of presence (Koechlin et al., 2003). Moreover, Jäncke et al. (2009) also remarked its importance in modulating and generating the activity of the network associated with the experience of presence. Regarding the positive correlations, we obtained significant activations in the lingual gyrus, cerebellum; middle, sub-gyral and superior temporal lobe; calcarine and cuneus. All of these areas are related to sense of presence, which explains why their activation gets higher along with the increase of the questionnaires scores. Particularly remarkable is the result for the lingual parahippocampal gyrus, more specifically the activation of the parahippocampal area, a sub-region of the parahippocampal cortex related to spatial orientation and encoding and recognition of scenes (Aguirre et al., 1996; Epstein and Kanwisher, 1998).

Referring to the parametric analysis, it showed a lineal trend between the three tasks associated with an increased feeling of presence in the insula and parietal lobe, two of the most significant areas we emphasized for the 'navigation > video' contrast, and which are related to self-awareness (Craig, 2009; Karnath et al., 2005) and navigation sense in a VE (Johnson et al., 1996; Mishkin and Ungerleider, 1982), respectively. The fact that these two areas showed a positive correlation with questionnaire scores, and in the parametric analysis, is an indicator of their relation to sense of presence.

To finish this discussion, we will address some of the limitations of our study. The study was conducted using a specific group of participants, namely 14 right-handed women. They were all right-handed to prevent noise effects of manual lateralization on brain activation in virtual/spatial processing. The subjects were all women to reduce variability generated by gender differences. There are some previous studies which show that women present a higher activation in the presence of emotional stimuli than men. In fact, Canli et al. (2001) indicated that they chose women because they respond more intensely to sensitive stimuli. They also maintained that women show a greater psychological reaction according to their value judgment than men. Some other studies concerning emotional arousal have also concluded that women demonstrate higher activation when shown disgusting images than when shown pleasant ones, while men do not demonstrate any difference (Lang et al., 1998). A great deal of previous studies concerning visual stimuli has been conducted with women (e.g., Dilger et al., 2003; Ochsner et al., 2002). Another limitation of our study was the small sample size, which restricts the statistical power to detect changes in the BOLD signal.

In our study, we added the continuous movement of the joystick to compensate the differences between experimental conditions in the activations caused by the motor tasks. However, there were differences in the active planning between the free navigation condition and the other two tasks, and these differences could not be prevented because they are one of the causes of the differences in the feeling of presence between experimental conditions. We should also remark as a limitation the low significance level we used for the statistical analysis of the fMRI data ( $p<0.001$ (uncorrected) may be a liberal threshold). Maybe the use of a $3 \mathrm{~T}$ scanner could improve the results obtained here.

\section{CONCLUSIONS}

In conclusion, the activation of the cuneus, the insula and the parietal areas should be noted, especially the latter, due to its relationship with the navigational aspects of the VR experience. As shown in Section 4, our final results are consistent with those 
from other studies concerning navigation in VR, presence in VR studied with other brain imaging techniques and presence during an automatic navigation in a VE studied with fMRI. Moreover, insula activation in VR and its parametric association with the sense of presence experienced in each of the conditions raises questions regarding its role in the virtual experience. However, the brain activation results may be seen just as a proof of the utility of fMRI as a tool to evaluate presence, and the important consequences that this could have in the field of the HCI. Although in this study we have generated differences in presence with changing navigation conditions, possible future research could involve more arousing environments, with different content, to analyze other factors that can induce presence. Moreover, the demonstration that presence is related to measurable differences in brain activity, even inside an unfriendly environment as it is an MR machine, opens the door to future studies combining VR with fMRI for psychological treatments and psychopathological applications.

\section{FUNDING}

This study was funded by the Ministerio de Educación y Ciencia Spain, Project Game Teen (TIN2010-20187) and partially by projects Consolider-C (SEJ2006-14301/PSIC), 'CIBER of Physiopathology of Obesity and Nutrition, an initiative of ISCIII', the Excellence Research Program PROMETEO (Generalitat Valenciana. Conselleria de Educación, 2008-157) and the Consolider INGENIO program (CSD2007-00012). The work of Miriam Clemente was supported by the Generalitat Valenciana under a VALi+d Grant.

\section{REFERENCES}

Aguirre, G.K., Detre, J.A., Alsop, D.C. and D'Esposito, M. (1996) The parahippocampus subserves topographical learning in man. Cereb. Cortex, 6, 823-829. doi:10.1093/cercor/6.6.823.

Alcañiz, M., Rey, B., Tembl, J. and Parkhutik, V. (2009) A neuroscience approach to virtual reality experience using transcranial Doppler monitoring. Presence, 18, 97-111. doi:10.1162/pres.18.2.97.

Amaro Jr, E. and Barker, G.J. (2006) Study design in fMRI: basic principles. Brain Cogn., 60, 220-232. doi:10.1016/j.bandc. 2005.11.009.

Astur, R.S., Germain, S.A., Baker, E.K., Calhoun, V., Pearlson, G.D. and Constable, R.T. (2005) fMRI hippocampal activity during a virtual radial arm maze. Appl. Psychophysiol. Biofeedback, 30, 307-317. doi: 10.1007/s10484-005-6385-z.

Baños, R.M., Botella, C., García-Palacios, A., Villa, H., Perpiñá, C. and Alcañiz, M. (2000) Presence and reality judgment in virtual environments: a unitary construct? Cyberpsychol. Behav., 3, 327335. doi:10.1089/10949310050078760.

Baumann, S., Neff, C., Fetzick, S., Stangl, G., Basler, L., Vereneck, R. and Schneider, W. (2003) A virtual reality system for neurobehavioral and functional MRI studies. Cyberpsychol. Behav., 6, 259-266. doi:10.1089/109493103322011542.

Baumgartner, T., Speck, D., Wettstein, D., Masnari, O., Beeli, G. and Jäncke, L. (2008) Feeling present in arousing virtual reality worlds: prefrontal brain regions differentially orchestrate presence experience in adults and children. Front. Hum. Neurosci., 2, 1-12. doi: 10.3389/neuro.09.008.2008.

Baumgartner, T., Valko, L., Esslen, M. and Jäncke, L. (2006). Neural correlate of spatial presence in an arousing and noninteractive virtual reality: an EEG and psychophysiology study. Cyberpsychol. Behav., 9, 30-45. doi:10.1089/cpb.2006.9.30.

Belliveau, J.W., Kennedy, D.N., McKinstry, R.C., Buchbinder, B.R., Weisskoff, R.M., Cohen, M.S., Vevea, J.M., Brady, T.J. and Rosen, B.R. (1991) Functional mapping of the human visual cortex by magnetic resonance imaging. Science, 254, 716-719. doi: 10.1126/science. 1948051 .

Born, R. and Bradley, D. (2005) Structure and function of visual area MT. Annu. Rev. Neurosci., 28, 157-89. doi:10.1146/ annurev.neuro.26.041002.131052.

Canli, T., Zhao, Z., Desmond, J.E., Kang, E., Gross, J. and Gabrieli, J.D.E. (2001) An fMRI study of personality influences on brain reactivity to emotional stimuli. Behav. Neurosci., 115, 33-42. doi: 10.1037/0735-7044.115.1.33.

Clemente, M., Rodríguez, A., Rey, B., Rodríguez, A., Baños, R.M., Botella, C., Alcañiz, M. and Ávila, C. (2011) Analyzing the level of presence while navigating in a virtual environment during an fMRI scan. P. Campos et al. (Eds.): INTERACT 2011, Part IV, Lect. Notes Comput. Sci. 6949, 475-478. doi: 10.1007/978-3-642-23768 $-3 \_61$.

Craig, A.D. (2009) How do you feel now? The anterior insula and human awareness. Nat. Rev. Neurosci., 10, 59-70. doi:10.1038/nrn2555.

Dilger, S., Straube, T., Mentzel, H.J., Fitzek, C., Reichenbach, J., Hecht, H., Krieschel, S., Gutberlet, I. and Miltner, W.H.R. (2003) Brain activation to phobia-related pictures in spider phobic humans: an event-related functional magnetic resonance imaging study. Neurosci. Lett., 348, 29-32. doi: 10.1016/S0304-3940(03) 00647-5.

Dillon, C., Keogh, E., Freeman, J., and Davidoff, J. (2000) Aroused and Immersed: The Psychophysiology of Presence. In Proceedings of 3rd International Workshop on Presence, Delft University of Technology, Delft, The Netherlands, 27-28 March 2000.

Dodds, C.M., Morein-Zamir, S. and Robbind, T.W. (2011) Dissociating inhibition, attention, and response control in the frontoparietal network using functional magnetic resonance imaging. Cereb. Cortex, 21, 1155-1165. doi: 10.1093/cercor/bhq187.

Epstein, R. and Kanwisher, N. (1998) A cortical representation of the local visual environment. Nature, 392, 598-601. doi: $10.1038 / 33402$.

Flach, J.M. and Holden, J.G. (1998) The reality of experience: Gibson's way. Presence-Teleop. Virt. Environ. 7, 90-95. doi:10.1162/105474698565550.

Friston, K.J., Holmes, A.P., Poline, J-B., Grasby, P.J., Williams, S.C.R., Frackowiak, R.S.J. and Turner, R. (1995) Analysis of 
fMRI time-series revisited. Neuroimage, 2, 45-53. doi:10.1006/ nimg.1995.1007.

Geake, J.G. and Hansen, P.C. (2005) Neural correlates of intelligence as revealed by fMRI of fluid analogies. NeuroImage, 26, 555-564. doi: 10.1016/j.neuroimage.2005.01.035.

Haans, A. and Ijsselsteijn, W.A. (2012) Embodiment and telepresence: towards an comprehensive theory of presence. Interact. Computers, 24, 211-218. doi:10.1016/j.intcom.2012.04.010.

Haldane, M., Cunningham, G., Androutsos, C. and Frangou, S. (2008) Structural brain correlates of response inhibition in bipolar disorder I. J. Psychopharmacol., 22, 138-143. doi: 10.1177/0269881107082955.

Hartley, T., Maguire, E.A., Spiers, H.J. and Burgess, N. (2003) The well-worn route and the path less traveled: distinct neural bases of route following and wayfinding in humans. Neuron, 37, 877-888. doi: 10.1016/S0896-6273(03)00095-3.

Heeter, C. (1992) Being there: the subjective experience of presence. Presence: Teleop. Virt. Environ., 1, 262-271.

International Society for Presence Research (2000) The concept of presence: explication statement. http://www.ispr.info/ (accessed October 28, 2011).

Jäncke, L., Cheetham, M. and Baumgartner, T. (2009) Virtual reality and the role of the prefrontal cortex in adults and children. Front. Neurosci., 3, 52-59. doi: 10.3389/neuro.01.006.20009.

Johnson, P.B., Ferraina, S., Bianchi, L. and Caminiti, R. (1996) Cortical networks for visual reaching: physiological and anatomical organization of frontal and parietal lobe arm regions. Cereb. Cortex, 6, 102-119. doi: 10.1093/cercor/6.2.102.

Karnath, H.O., Baier, B. and Nägele, T. (2005) Awareness of the functioning of one's own limbs mediated by the insular cortex? J. Neurosci., 25, 7134-7138. doi: 10.1523/?JNEUROSCI.159005.2005 .

Kim, T. and Biocca, F. (2006) Telepresence via television: two dimensions of telepresence may have different connections to memory and persuasion. J. Comput. Mediated Commun., 3. doi: 10.1111/j.1083-6101.1997.tb00073.x.

Koechlin, E., Ody, C. and Kouneiher, F. (2003) The architecture of cognitive control in the human prefrontal cortex. Science, 302, 1181-1185. doi: 10.1126/science.1088545.

Lang, P.J., Bradley, M.M., Fitzsimmons, J., Cuthbert, B.N., Scott, J.D., Moulder, B. and Nangia, V. (1998) Emotional arousal and activation of the visual cortex: an fMRI analysis. Psychophysiology, 35, 199-210. doi: 10.1111/1469-8986.3520199.

Le Bihan, D., Turner, R., Zeffiro, T.A., Cuénod, C.A., Jezzard, P. and Bonnerot, V. (1993) Activation of human primary visual cortex during visual recall: a magnetic resonance imaging study. Proc. Natl. Sci. USA, 90, 11802-11805.

Lessiter, J., Freeman, J., Keogh, E. and Davidoff, J. (2001) A cross-media presence questionnaire: the ITC-sense of presence inventory. Presence-Teleop. Virt. Environ., 10, 282-298. doi:10.1162/105474601300343612.

Lombard, M. and Ditton, T. (2006) At the heart of it all: the concept of presence. J. Comput. Mediated Commun. 3. doi: 10.1111/j.10836101.1997.tb00072.x.
Loomis, J.M. (1992) Distal attribution and presence. Presence, 1, 113-118.

Meehan, M., Insko, B., Whitton, M. and Brooks, F.P. Jr. (2001) Physiological measures of presence in virtual environments. In Proceedings of 4th International Workshop on Presence, Philadelphia, USA, 21-23 May 2001.

Mellet, E., Laou, L., Petit, L., Zago, L., Mazoyer, B. and TzourioMazoyer, N. (2010) Impact of the virtual reality on the neural representations of an environment. Hum. Brain Mapp., 31, 1065-1075. doi: 10.1002/hbm.20917.

Mishkin, M. and Ungerleider, L.G. (1982) Contribution of striate inputs to the visuospatial functions of parieto-preoccipital cortex in monkeys. Behav. Brain Res., 6, 57-77. doi: 10.1016/01664328(82)90081-X.

Mraz, R., Hong, J., Quintin, G., Staines, W.R., McIlroy, W.E., Zakzanis, K.K. and Graham, S.J. (2003) A platform for combining virtual reality experiments with functional magnetic resonance imaging. Cyberpsychol. Behav., 6, 359-368. doi:10.1089/109493103322278736.

Ochsner, K.N., Bunge, S.A., Gross, J.J. and Gabrieli, J.D.E. (2002) Rethinking feelings: an fMRI study of the cognitive regulation of emotion. J. Cogn. Neurosci., 14, 1215-1229. doi: $10.1162 / 089892902760807212$.

Oldfield, R.C. (1971) The assessment and analysis of handedness: the Edinburgh inventory. Neuropsychologia, 9, 97-113. doi:10.1016/0028-3932(71)90067-4.

Owen, A.M., Downes, J.J., Sahakian, B.J., Polkey, C.E. and Robbins, T.W. (1990) Planning and spatial working memory following frontal lobe lesions in man. Neuropsychologia, 28, 1021-1034. doi: 10.1016/0028-3932(90)90137-D.

Perani, D., Fazio, F., Borghese, N.A., Tettamanti, M., Ferrari, S., Decety, J. and Gilardi, M.C. (2001) Different brain correlates for watching real and virtual hand actions. NeuroImage, 14, 749-758. doi: 10.1006/nimg.2001.0872.

Petrides, M. (2000) The role of the mid-dorsolateral prefrontal cortex in working memory. Exp. Brain Res., 133, 44-54. doi: $10.1007 / \mathrm{s} 002210000399$.

Pine, D.S., Grun, J., Maguire, E.A., Burgess, N., Zarahn, E., Koda, V., Fyer, A., Szeszko, P.R. and Bilder, R.M. (2002) Neurodevelopmental aspects of spatial navigation: a virtual reality fMRI study. NeuroImage, 15, 396-406. doi: 10.1006/nimg.2001.0988.

Riva, G., Davide, F. and Ijsselsteijn, W.A. (2003) Being There: Concepts, Effects and Measurement of User Presence in Synthetic Environments. IOS Press, Amsterdam, The Netherlands.

Riva, G., Waterworth, J.A., Waterworth, E.L. and Mantovani, F. (2011) From intention to action: the role of presence. New Ideas Psychol., 29, 24-37. doi:10.1016/j.newideapsych.2009.11.002.

Rey, B., Alcañiz, M., Tembl, J. and Parkhutik, V. (2010) Brain activity and presence: a preliminary study in different immersive conditions using transcranial Doppler monitoring. Virt. Real., 14, 55-65. doi: 10.1007/s10055-009-0141-2.

Sanchez-Vives, M.V. and Slater, M. (2005) From presence to consciousness through virtual reality. Nat. Rev. Neurosci., 6, 332339. doi:10.1038/nrn1651. 
Sadowski, W. and Stanney, K. (2002) Presence in virtual environments. In K. Stanney (ed.), Handbook of Virtual Environments: Design, Implementation, and Applications, pp. 791-806. Lawrence Erlbaum Associates, New Jersey.

Scheibe, C., Wartenburger, I., Wüstenberg, T., Kathmann, N., Villringer, A. and Heekeren, H.R. (2006) Neural correlates of the interaction between transient and sustained processes: a mixed blocked/event-related fMRI study. Hum. Brain Mapp., 27, 545-551. doi: 10.1002/hbm.20199.

Schuemie, M.J., Van Der Straaten, P., Krijn, M. and Van Der Mast, C.A.P.G. (2001) Research on presence in virtual reality: a survey. Cyberpsychol. Behav., 4, 183-201. doi:10.1089/ 109493101300117884.

Sharp, H., Rogers, Y. and Preece, J. (2007) Interaction Design: Beyond Human-Computer Interaction, 2nd ed. Wiley, New York.

Sheridan, T.B. (1992) Musings on telepresence and virtual presence. Presence-Teleop. Virt. Environ., 1, 120-126.

Sjölie, D. (2012) Presence and general principles of brain function. Interact. Comput. doi:10.1016/j.intcom.2012.04.004

Sjölie, D., Bodin, K., Elgh, E., Eriksson, J., Janlert, L.-E. and Nyberg, L. (2010) Effects of interactivity and 3D-motion on mental rotation brain activity in an immersive virtual environment. In Proceedings of the 28th International Conference on Human Factors in Computing Systems, pp. 869-878. ACM, Atlanta, GA.

Slater, M. and Wilbur, M. (1997) A framework for immersive virtual environments (FIVE) - speculations on the role of presence in virtual environments. Presence-Teleop. Virt. Environ., 6, 603-616.

Smith, S.M. (2004) Overview of fMRI analysis. Br. J. Radiol., 77, 167-175. doi: 10.1259/bjr/33553595.
Usoh, M., Catena, E., Arman, S. and Slater, M. (2000) Using presence questionnaires in reality. Presence-Teleop. Virt. Environ., 9, 497503. doi:10.1162/105474600566989.

Vanni, S., Tanskanen, T., Seppä, M., Uutela, K. and Hari, R. (2001) Coinciding early activation of the human primary visual cortex and anteromedial cuneus. Proc. Natl Acad. Sci. USA, 98, 2776-2780. doi: 10.1073/pnas.041600898.

Waterworth, J. A., Waterworth, E. L. and Westling, J. (2002) Presence as performance: the mystique of digital participation. In Proceedings of 5th Annual International Workshop on Presence, Porto, Portugal, October 2002.

Welch, R., Blackmon, T., Liu, A., Mellers, B. and Stark, L. (1996) The effects of pictorial realism, delay of visual feedback, and observer interactivity on the subjective sense of presence. Presence-Teleop. Virt. Environ., 5, 263-273.

Willshaw, D. (1999) The cerebellum as a neuronal machine. In Proceedings of Self-Learning Robots III Brainstyle Robotics: The Cerebellum Beyond Function Approximation (Ref. No. 1999/049), IEE Workshop, London, UK, 3-1 January 1999. doi: 10.1049/ic: 19990258

Witmer, B.G. and Singer, M.J. (1998) Measuring presence in virtual environments: a presence questionnaire. Presence-Teleop. Virt. Environ., 7, 225-240. doi:10.1162/105474698565686.

Wolf, U., Rapoport, M.J. and Schweizer, T.A. (2009) Evaluating the affective component of the cerebellar cognitive affective syndrome. J. Neuropsychiatry Clin. Neurosci., 21, 245-253. doi: 10.1176/appi.neuropsych.21.3.245.

Zahorik, P. and Jenison, R.L. (1998) Presence as being-in-theworld. Presence-Teleop. Virt. Environ., 7, 78-89. doi:10.1162/ 105474698565541. 